\title{
Coexistence in a two-type continuum growth model
}

\author{
Maria Deijfen * Olle Häggström ${ }^{\dagger}$
}

August 2004

\begin{abstract}
We consider a stochastic model, describing the growth of two competing infections on $\mathbb{R}^{d}$. The growth takes place by way of spherical outbursts in the infected region, an outburst in the type 1 (2) infected region causing all previously uninfected points within a stochastic distance from the outburst location to be type 1 (2) infected. The main result is that, if the infection types have the same intensity, then there is a strictly positive probability that both infection types grow unboundedly.
\end{abstract}

Keywords: Richardson's model, competing growth, coexistence.

AMS 2000 Subject Classification: Primary 60K35

Secondary 82B43.

\section{Introduction}

In Deijfen (2003), a growth model is introduced that describes the spread of an entity, henceforth referred to as an infection, on $\mathbb{R}^{d}$. The model is generalized in Deijfen et al (2003) to encompass two infection types, making it a model for competition on $\mathbb{R}^{d}$. Deijfen et al (2003) is mainly concerned with the case when the infections have unequal intensities and the main result is roughly that two infections with different intensities cannot simultaneously grow to occupy infinite parts of $\mathbb{R}^{d}$. In this paper we consider infections with

*Stockholm University. E-mail: mia@matematik.su.se

${ }^{\dagger}$ Chalmers University of Technology. E-mail: olleh@math.chalmers.se 
the same intensity and show that in this case there is a positive probability that both infection types reach points arbitrarily far from the origin.

The Richardson model, introduced in Richardson (1973), describes growth on $\mathbb{Z}^{d}$. Sites are either healthy or infected, the dynamics being that a healthy site is infected at a rate proportional to the number of infected neighbors and once infected it remains so forever. The model of Deijfen (2003) can be viewed as a kind of continuum analog of the Richardson model. Starting from an arbitrary initial set, the growth is driven by randomly sized spherical outbursts in the infected region, whose value at time $t$ is denoted by $S_{t}$. The time until an outburst takes place is exponentially distributed with parameter proportional to $\left|S_{t}\right|$, where $|\cdot|$ denotes Lebesgue measure, and the outburst point is uniformly distributed over $S_{t}$. When an outburst occurs it causes the previously uninfected parts of a ball centered at the outburst point to be infected, the radii in the outburst balls being i.i.d. random variables with distribution $F$. The main result for the model is a shape theorem stating that, if

$$
\int_{0}^{\infty} e^{-\varphi r} d F(r)<\infty \text { for some } \varphi<0,
$$

then, on the scale $1 / t$, the set $S_{t}$ has an asymptotic shape, which by rotational invariance is forced to be a Euclidean ball; see Theorem 1.1 in Deijfen et al (2003). This is a stronger result than for the Richardson model, for which the existence of an asymptotic shape is established but the nature of the shape so far remains unknown.

In Häggström and Pemantle $(1998,2000)$ a generalized version of the Richardson model is introduced that contains two infection types, referred to as type 1 and type 2 infection respectively. The dynamics is that, for $i=1,2$, a healthy site becomes type $i$ infected at rate $\lambda_{i}$ times the number of type $i$ infected nearest neighbors and once infected it never recovers. Here $\lambda_{i} \in \mathbb{R}$ indicates the intensity of the type $i$ infection. Furthermore, when a site is struck by one of the infection types, it is assumed to become immune to the other type, making the process a model for competing growth on $\mathbb{Z}^{d}$.

The continuum model of Deijfen (2003) can also be extended to incorporate two infection types; this is done in Deijfen et al (2003). Like in the one-type model, the growth takes place by way of spherical outbursts, the type of an outburst being determined by the region in which it takes place. Let $S_{t}^{1}\left(S_{t}^{2}\right)$ denote the region occupied by type $1(2)$ infection at time $t$. The time until an outburst occurs in $S_{t}^{i}(i=1,2)$ is exponentially distributed 
with parameter $\lambda_{i}\left|S_{t}^{i}\right|$, where $\lambda_{i} \in \mathbb{R}$ is the intensity of the type $i$ infection, and the outburst point is chosen uniformly in $S_{t}^{i}$. An outburst in the type $i$ infected region hurls out type $i$ infection to all previously uninfected points in a ball centered at the outburst point. The radii in the outburst balls are i.i.d. random variables with the same distribution, denoted by $F$, for both outburst types. Note that the infection types are assumed to be mutually exclusive, that is, a point can be contained in at most one of the sets $S_{t}^{1}$ and $S_{t}^{2}$.

According to the above mechanisms, the two-type models describe a competition for space - on $\mathbb{Z}^{d}$ and $\mathbb{R}^{d}$ respectively - between two infections. For both models there are three possible results of this competition: Either one of the infection types wins or there is a draw. For $i=1,2$, define

$$
A_{i}=\{\text { the type } i \text { infection reaches sites arbitrarily far from the origin }\} .
$$

We say that the type 1 (2) infection has won if $\neg A_{2}\left(\neg A_{1}\right)$ occurs and with a draw we mean that $A=A_{1} \cap A_{2}$ occurs, that is, a power balance is established so that both infection types keep growing indefinitely; here $\neg$ denotes set complement.

In the discrete model and in the continuum model with bounded outburst radii, clearly both events $\neg A_{1}$ and $\neg A_{2}$ - and thereby also the events $A_{1}$ and $A_{2}$ - have positive probability regardless of the values of the intensities $\lambda_{1}$ and $\lambda_{2}$. In the continuum model with unbounded support for the outburst radii, this fact requires a proof; see Proposition 5.1 in Deijfen et al (2003). The mathematical challenge with the two-type models lies in deciding whether the event $A$ has positive probability or not. Intuitively, the state of equilibrium represented by $A$ should be possible to maintain in the long run if and only if the infection types have the same intensity. To state this conjecture more formally, let $\tilde{P}^{\lambda_{1}, \lambda_{2}}$ denote the probability law for a discrete two-type process started from two single nodes, one at the origin and one at the point $(1,0, \ldots, 0)$, and write $P^{\lambda_{1}, \lambda_{2}}$ for the distribution of a continuum two-type process started from two unit balls located next to each other, one centered at the origin and the other at the point $(2,0, \ldots, 0)$.

Conjecture 1.1 For any dimension $d \geq 2$, we have

(a) $\tilde{P}^{\lambda_{1}, \lambda_{2}}(A)>0 \Leftrightarrow \lambda_{1}=\lambda_{2}$;

(b) if F satisfies (11), then $P^{\lambda_{1}, \lambda_{2}}(A)>0 \Leftrightarrow \lambda_{1}=\lambda_{2}$. 
Remark 1.1 It is shown in Deijfen and Häggström (2003) that the possibility of infinite coexistence in the lattice model is not affected by the initial configuration, that is, whether $\tilde{P}^{\lambda_{1}, \lambda_{2}}(A)$ is positive or not does not depend on the initial state of the model, given of course that neither of the infection types surrounds the other already at the beginning. In Deijfen et al (2003) an analogous result (stated as Proposition 3.1 in the present paper) is proved for the continuum model provided the technical condition

$$
F(\varepsilon)>0 \text { for all } \varepsilon>0 \text {. }
$$

Hence the particular choice of initial sets is unimportant in Conjecture 1.1.

Efforts have been made to prove Conjecture 1.1 and a number of partial results have been obtained. As for the case with unequal intensities, it is shown in Häggström and Pemantle (2000) that, if $\lambda_{1}$ is fixed, then $\tilde{P}^{\lambda_{1}, \lambda_{2}}(A)=0$ for all but at most countably many values of $\lambda_{2}$, and in Deijfen et al (2003) the same result is established for $P^{\lambda_{1}, \lambda_{2}}$ under the assumption that (11) holds for $F$. Unfortunately, to strengthen these results to cover all intensities $\lambda_{2} \neq \lambda_{1}$ seems to be marred by large technical difficulties.

The discrete model with equal intensities was first studied by Häggström and Pemantle (1998). Their main result is that, if $\lambda_{1}=\lambda_{2}$ and $d=2$, then $\tilde{P}^{\lambda_{1}, \lambda_{2}}(A)>0$, that is, on a two-dimensional square lattice, mutual unbounded growth is possible for infections with the same intensity. Recently Garet and Marchand (2004) were able to extend this result to arbitrary dimensions. While the technique of Häggström and Pemantle relies on some very specific features of the square lattice $\mathbb{Z}^{2}$, the proof of Garet and Marchand is much less sensitive to the exact lattice structure, and thus more amenable to generalizations. In this paper, we exploit the ideas of Garet and Marchand to establish infinite coexistence in the continuum setting. That is, we prove the following.

Theorem 1.1 Let $d \geq 2$ and assume that $F$ satisfies conditions (11) and (2). Then, if $\lambda_{1}=\lambda_{2}$, we have $P^{\lambda_{1}, \lambda_{2}}(A)>0$.

Remark 1.2 Condition (2), which says that arbitrarily small outbursts happen with positive probability, is only needed in order to apply Proposition 3.1 below (quoted from Deijfen et al (2003)). Without assuming (2), we still have the slightly weaker result that infinite coexistence has positive probability for certain other compactly supported initial states - see Theorem 
3.1. Furthermore, we strongly believe that Proposition 3.1 holds without the condition (2).

The most important step we need to take in order to be able to invoke the Garet-Marchand ideas is a new coupling construction of the two-type continuum model, designed in such a way that certain subadditivity properties of the process are reflected in the coupling. This construction is described in Section 2 together with some preliminary results, and the proof of Theorem 1.1 is given in Section 3. Superficially, our proof may not seem to have much in common with the Garet-Marchand paper, but the underlying key idea is the same. Translated to the continuum setting, the basic idea is to observe (17) and to demonstrate that $P^{\lambda_{1}, \lambda_{2}}(A)=0$ would lead to consequences that contradict (7).

\section{A new coupling construction}

Let us begin by describing in more detail how the continuum two-type model with equally powerful infections can be generated. To be able to exploit the Garet-Marchand ideas, we need to modify the construction in Deijfen (2003) and Deijfen et al (2003) in order to make a certain subbadditivity relation (41) hold.

To construct the model in $d$ dimensions, consider a Poisson process on $\mathbb{R}^{d+1}$ with rate $\lambda$, where the extra dimension represents time and $\lambda$ denotes the common intensity of the infection types. Associate independently to each Poisson point a random radius variable with distribution $F$ and pick two bounded disjoint regions $S_{0}^{1}, S_{0}^{2} \subset \mathbb{R}^{d}$ to start up the growth, the set $S_{0}^{i}$ $(i=1,2)$ indicating the initially type $i$ infected region.

Starting at time 0 , the growth is now brought about by scanning within the region $\left(S_{0}^{1} \cup S_{0}^{2}\right) \times \mathbb{R}$ upwards along the time axis until a point in the Poisson process is hit. An outburst then occurs at this point, causing the previously uninfected parts of a ball around the outburst point to be infected. The radius of the outburst ball is given by the radius variable associated with the Poisson point and the type of the transmitted infection is determined by in which one of the regions $S_{0}^{1} \times \mathbb{R}$ and $S_{0}^{2} \times \mathbb{R}$ the Poisson point is found. Let $T_{1}$ denote the time at which this first outburst takes place and, for $i=1,2$, let $S_{(1)}^{i}$ denote the type $i$ infected region after the outburst. Here the region with the same type as the outburst might be enlarged as compared to before the outburst, while the other region remains unchanged. 
To generate the next outburst, we keep scanning upwards in time for Poisson points, but in such a way that the region $S_{0}^{1} \cup S_{0}^{2}$ and the region $\left(S_{(1)}^{1} \cup S_{(1)}^{2}\right) \backslash$ $\left(S_{0}^{1} \cup S_{0}^{2}\right)$ are treated somewhat differently: for the region $S_{0}^{1} \cup S_{0}^{2}$, we scan from time $T_{1}$ and upwards, while simultaneously for $\left(S_{(1)}^{1} \cup S_{(1)}^{2}\right) \backslash\left(S_{0}^{1} \cup S_{0}^{2}\right)$, we scan from time 0 and upwards. Hence, the time $T_{2}$ of the next outburst is given by the minimum of $T_{2}^{\prime}$ and $T_{2}^{\prime \prime}$, where $T_{2}^{\prime}$ is the time coordinate of the second Poisson point in $\left(S_{0}^{1} \cup S_{0}^{2}\right) \times \mathbb{R}$, and $T_{2}^{\prime \prime}$ is the sum of $T_{1}$ and the time coordinate of the first Poisson point in $\left(S_{(1)}^{1} \cup S_{(1)}^{2}\right) \backslash\left(S_{0}^{1} \cup S_{0}^{2}\right) \times \mathbb{R}$.

Subsequent outbursts are generated by similarly scanning up the time axis. This means that a Poisson point at coordinates $(x, t)$ with $x \in \mathbb{R}^{d}$ and $t>0$ represents an outburst at $x$, not at (absolute) time $t$, but precisely $t$ time units after the point $x$ first became infected. We thus obtain two increasing sequences $\left\{S_{(n)}^{1}\right\}$ and $\left\{S_{(n)}^{2}\right\}$ of regions in $\mathbb{R}^{d}$ indicating the type 1 and type 2 infected regions after $n$ outbursts, and a strictly increasing sequence $\left\{T_{n}\right\}$ specifying the time points for the outbursts. For $t \in\left[T_{n}, T_{n+1}\right)$, the type $i$ infected region at time $t$ is given by $S_{t}^{i}=S_{(n)}^{i}$ and the total infected region at time $t$ is $S_{t}=S_{t}^{1} \cup S_{t}^{2}$. Clearly this defines a model where the time until an outburst occurs in $S_{i}^{t}$ is exponentially distributed with parameter $\lambda\left|S_{t}^{i}\right|$ and the location of the outburst is uniformly distributed in $S_{t}^{i}$, as desired.

In summary, the probability space underlying our construction, is simply a marked Poisson process on $\mathbb{R}^{d+1}$ with rate $\lambda$ and i.i.d. marks with distribution $F$. A more thorough description of the two-type model can be found in Section 3 in Deijfen et al (2003). The construction there is different from the one given here, but it is easy to see that, if the infection types have the same intensity, the two construction methods result in growth processes with the same distribution. The difference between the two constructions matter only when we couple realizations of processes starting from different initial configurations.

If we do not distinguish between the type 1 and the type 2 infection, then the symmetric two-type model reduces to the one-type model of Deijfen (2003), that is, the total infected region in the two-type model behaves like a one-type process. An important consequence of this is the existence of a so called time constant, denoted by $\mu$, indicating the inverse asymptotic speed of the growth. Let $B(x, r)$ denote the closed ball with radius $r$ centered at $x \in \mathbb{R}^{d}$, and, for $x, y \in \mathbb{R}^{d}$, let $T_{x, y}$ denote the time when the ball $B(y, 1)$ is fully infected in a one-type process with rate $\lambda$ started with $S_{0}=B(x, 1)$. Also, write $\mathbf{n}=(n, 0, \ldots, 0)$. If the radius distribution $F$ satisfies (1), we have 


$$
\lim _{n \rightarrow \infty} \frac{T_{\mathbf{0 , n}}}{n}=\lambda^{-1} \mu \quad \text { a.s. and in } L^{1},
$$

and the same limit is obtained for the time when the single point $\mathbf{n}$ is infected; see Deijfen et al (2003) or note that, with the modified construction of the process, (3) follows immediately from Liggett's subadditive ergodic theorem. The reason for considering the time when the entire unit ball around the target point is infected (rather than the time at which the point itself is infected) is that, in combination with the particular way the model is constructed from the Poisson process, it gives rise to the following very useful subadditivity property.

Lemma 2.1 For any $x, y, z \in \mathbb{R}^{d}$, we have

$$
T_{x, y} \leq T_{x, z}+T_{z, y} .
$$

Proof: For $t \geq 0$, let $S_{t}$ denote the infected region at time $t$ starting with $S_{0}=B(x, 1)$, and let $\tilde{S}_{t}$ denote the infected region at time $t$ starting with $\tilde{S}_{0}=B(z, 1)$. We need to show that

$$
B(y, 1) \subseteq S_{T_{x, z}+T_{z, y}} .
$$

By definition of $T_{z, y}$, we have that $B(y, 1) \subseteq \tilde{S}_{T_{z, y}}$. Hence it suffices to show that, for any $t \geq 0$, we have

$$
\tilde{S}_{t} \subseteq S_{T_{x, z}+t} .
$$

For $t=0$, (6) is immediate from the definition of $T_{x, z}$. It remains to show that the set inclusion is preserved as $t$ increases. The only $t$ 's for which it could possibly stop holding are at the times of outbursts in the $\tilde{S}$-process. Consider the first such outburst at which (6) ceases to hold, denote the time (in the $\tilde{S}$-process) at which this outburst happens by $t^{\prime}$, and denote its location by $x$. Since (6) holds for all $t<t^{\prime}$, we have that at time $T_{x, z}+t^{\prime}$ the point $x$ has been infected in the $S$-process for at least as long as it has been infected in the $\tilde{S}$-process at time $t^{\prime}$. It therefore follows from the construction of the process that the $S$-process by time $T_{x, z}+t^{\prime}$ has already had an outbreak at the point $x$ (or has such an outbreak precisely at time $T_{x, z}+t^{\prime}$ ) of the same radius as the outbreak at $x$ in the $\tilde{S}$-process. Hence, (6) never ceases to hold and consequently holds for all $t$. In particular, taking $t=T_{z, y}$ yields (5), and we are done.

Using the subadditivity in Lemma 2.1, let us prove the following lemma which will be crucial in the proof of Theorem 1.1 . 
Lemma 2.2 We have

$$
\limsup _{m \rightarrow \infty} \mathrm{E}\left[T_{\mathbf{n},-\mathbf{m}}-T_{\mathbf{0},-\mathbf{m}}\right] \geq n \lambda^{-1} \mu .
$$

Proof: Note first that, since $\mathrm{E}\left[T_{\mathbf{n},-\mathbf{m}}\right]=\mathrm{E}\left[T_{\mathbf{0}, \mathbf{n}+\mathbf{m}}\right]$ and $\mathrm{E}\left[T_{\mathbf{0},-\mathbf{m}}\right]=\mathrm{E}\left[T_{\mathbf{0}, \mathbf{m}}\right]$, we have

$$
\mathrm{E}\left[T_{\mathbf{n},-\mathbf{m}}-T_{\mathbf{0},-\mathbf{m}}\right]=\mathrm{E}\left[T_{\mathbf{0}, \mathbf{n}+\mathbf{m}}-T_{\mathbf{0}, \mathbf{m}}\right] .
$$

Hence we are done if we can show that

$$
\limsup _{m \rightarrow \infty} \mathrm{E}\left[T_{\mathbf{0}, \mathbf{n}+\mathbf{m}}-T_{\mathbf{0}, \mathbf{m}}\right] \geq n \lambda^{-1} \mu .
$$

To do this, note that, for any positive integer $k, \mathrm{E}\left[T_{\mathbf{0}, k \mathbf{n}}\right]$ can be rewritten as the telescoping sum

$$
\mathrm{E}\left[T_{\mathbf{0}, k \mathbf{n}}\right]=\sum_{i=1}^{k} \mathrm{E}\left[T_{\mathbf{0}, i \mathbf{n}}-T_{\mathbf{0},(i-1) \mathbf{n}}\right] .
$$

Suppose now for contradiction that (8) fails. Then there exists an $\varepsilon>0$ such that $\mathrm{E}\left[T_{\mathbf{0}, i \mathbf{n}}-T_{\mathbf{0},(i-1) \mathbf{n}}\right]<n \lambda^{-1} \mu-\varepsilon$ for all but at most finitely many positive integers $i$. Using (9), this implies that

$$
\limsup _{k \rightarrow \infty} \frac{\mathrm{E}\left[T_{\mathbf{0}, k \mathbf{n}}\right]}{k} \leq n \lambda^{-1} \mu-\varepsilon
$$

but according to (3) we have

$$
\lim _{k \rightarrow \infty} \frac{\mathrm{E}\left[T_{\mathbf{0}, k \mathbf{n}}\right]}{k}=n \lambda^{-1} \mu .
$$

Comparing (10) and (111) yields the desired contradiction, and the lemma is proved.

\section{Proof of Theorem 1.1}

First we quote a proposition that states that the initial configuration is basically irrelevant for the possibility of mutual unbounded growth in the two-type continuum model. Here $P_{\Gamma_{1}, \Gamma_{2}}^{\lambda_{1}, \lambda_{2}}$ denotes the probability law of a process started with $S_{0}^{1}=\Gamma_{1}$ and $S_{0}^{2}=\Gamma_{2}$. 
Proposition 3.1 (Deijfen et al 2003) Let $\left(\Gamma_{1}, \Gamma_{2}\right)$ and $\left(\Gamma_{1}^{\prime}, \Gamma_{2}^{\prime}\right)$ be two pairs of disjoint, bounded subsets of $\mathbb{R}^{d}$ with strictly positive Lebesgue measures. Furthermore, suppose that the radius distribution $F$ has unbounded support and satisfies $F(\varepsilon)>0$ for all $\varepsilon>0$. Then

$$
P_{\Gamma_{1}, \Gamma_{2}}^{\lambda_{1}, \lambda_{2}}(A)>0 \Rightarrow P_{\Gamma_{1}^{\prime}, \Gamma_{2}^{\prime}}^{\lambda_{1}, \lambda_{2}}(A)>0 .
$$

Remark 3.1 Proposition 3.1 extends to the case with bounded support as well, provided the following (obviously necessary) condition on $\left(\Gamma_{1}^{\prime}, \Gamma_{2}^{\prime}\right)$ : If the radius distribution is bounded by $r$, then neither of $\Gamma_{1}^{\prime}$ or $\Gamma_{2}^{\prime}$ may contain an impenetrable layer of thickness $r$ around the other.

Now, to prove Theorem 1.1 we will show that infinite coexistence is indeed possible for two infections with the same intensity if the initial sets are located sufficiently far away from each other; see Theorem 3.1. In view of the above proposition, clearly this implies Theorem 1.1. To simplify notation, write $P_{B(\mathbf{0}, 1), B(\mathbf{n}, 1)}^{\lambda_{1}, \lambda_{2}}=P_{\mathbf{0}, \mathbf{n}}^{\lambda_{1}, \lambda_{2}}$.

Theorem 3.1 If $F$ satisfies (1) and $\lambda_{1}=\lambda_{2}$, then $P_{\mathbf{0}, \mathbf{n}}^{\lambda_{1}, \lambda_{2}}(G)>0$ for large $n$.

Proof: By time-scaling, we may without loss of generality assume that $\lambda_{1}=$ $\lambda_{2}=1$. Hence, in what follows, only unit rate processes are considered. To avoid superfluous use of superscripts, write $P_{\mathbf{0}, \mathbf{n}}^{1,1}=P_{\mathbf{0}, \mathbf{n}}$.

First, fix $\varepsilon>0$ and pick $n$ large so that

(i) $\mathrm{E}\left[T_{\mathbf{0}, \mathbf{n}}\right] \leq(1+\varepsilon) n \mu$;

(ii) $P_{\mathbf{0 , n}}\left(T_{\mathbf{0}, \mathbf{n}}<(1-\varepsilon) n \mu\right)<\varepsilon$,

which is possible because of (3). The symbol 'E' will throughout the proof be used to denote expected value with respect to the marked Poisson process underlying the coupling construction described in Section 2. Now, by Lemma 2.2, there exist arbitrarily large $m$ such that

$$
\mathrm{E}\left[T_{\mathbf{n},-\mathbf{m}}-T_{\mathbf{0},-\mathbf{m}}\right] \geq(1-\varepsilon) n \mu .
$$

We will show that, if $P_{\mathbf{0}, \mathbf{n}}(A)=0$ and $\varepsilon$ is small, then (12) fails for large $m$, implying that we must have $P_{\mathbf{0}, \mathbf{n}}(A)>0$.

Denote by $B_{k}$ a ball with radius $k$ centered at the point $\mathbf{n} / 2$ and, for a two-type process started with $S_{0}^{1}=B(\mathbf{0}, 1)$ and $S_{0}^{2}=B(\mathbf{n}, 1)$, let

$$
A_{k}^{i}=\left\{\text { the type } i \text { infection reaches points outside } B_{k}\right\} \text {. }
$$


Also, define $A_{k}=A_{k}^{1} \cap A_{k}^{2}$, that is, $A_{k}$ is the event that both infection types reach points outside $B_{k}$. Note that, since $A=\cap_{k=1}^{\infty} A_{k}$ and $A_{k} \supset A_{k+1}$, we have

$$
P_{\mathbf{0}, \mathbf{n}}(A)=\lim _{k \rightarrow \infty} P_{\mathbf{0}, \mathbf{n}}\left(A_{k}\right) .
$$

Now assume that $P_{\mathbf{0}, \mathbf{n}}(A)=0$ and pick $m$ large enough so that $P_{\mathbf{0}, \mathbf{n}}\left(A_{m}\right)<$ $1 / 2$. Trivially,

$$
\begin{aligned}
\mathrm{E}\left[T_{\mathbf{n},-\mathbf{m}}-T_{\mathbf{0},-\mathbf{m}}\right] & =\mathrm{E}\left[\mathbf{1}_{\left\{A_{m}^{1}\right\}}\left(T_{\mathbf{n},-\mathbf{m}}-T_{\mathbf{0},-\mathbf{m}}\right)\right] \\
& +\mathrm{E}\left[\mathbf{1}_{\left\{\neg A_{m}^{1}\right\}}\left(T_{\mathbf{n},-\mathbf{m}}-T_{\mathbf{0},-\mathbf{m}}\right)\right],
\end{aligned}
$$

where $\mathbf{1}_{\{\cdot\}}$ denotes the indicator function. On $\neg A_{m}^{1}$, we have $T_{\mathbf{n},-\mathbf{m}}-$ $T_{\mathbf{0},-\mathbf{m}}<0$, and hence

$$
\begin{aligned}
\mathrm{E}\left[T_{\mathbf{n},-\mathbf{m}}-T_{\mathbf{0},-\mathbf{m}}\right] & \leq \mathrm{E}\left[\mathbf{1}_{\left\{A_{m}^{1}\right\}}\left(T_{\mathbf{n},-\mathbf{m}}-T_{\mathbf{0},-\mathbf{m}}\right)\right] \\
& \leq \mathrm{E}\left[\mathbf{1}_{\left\{A_{m}^{1}\right\}} T_{\mathbf{0}, \mathbf{n}}\right]
\end{aligned}
$$

where the last inequality follows from Lemma 2.1. To bound the right hand side from above, write

$$
\mathrm{E}\left[\mathbf{1}_{\left\{A_{m}^{1}\right\}} T_{\mathbf{0}, \mathbf{n}}\right]=\mathrm{E}\left[T_{\mathbf{0}, \mathbf{n}}\right]-\mathrm{E}\left[\mathbf{1}_{\left\{\neg A_{m}^{1}\right\}} T_{\mathbf{0}, \mathbf{n}}\right] .
$$

By the choice of $n$, we have $\mathrm{E}\left[T_{\mathbf{0}, \mathbf{n}}\right] \leq(1+\varepsilon) n \mu$, and so it remains to find a lower bound for $\mathrm{E}\left[\mathbf{1}_{\left\{\neg A_{m}^{1}\right\}} T_{\mathbf{0}, \mathbf{n}}\right]$. To this end, remember that, also by the choice of $n$, we have $P_{\mathbf{0}, \mathbf{n}}\left(T_{\mathbf{0}, \mathbf{n}}<(1-\varepsilon) n \mu\right)<\varepsilon$. Hence

$$
\begin{aligned}
\mathrm{E}\left[\mathbf{1}_{\left\{\neg A_{m}^{1}\right\}} T_{\mathbf{0}, \mathbf{n}}\right] & \geq P_{\mathbf{0}, \mathbf{n}}\left(\neg A_{m}^{1} \cap\left\{T_{\mathbf{0}, \mathbf{n}} \geq(1-\varepsilon) n \mu\right\}\right) \cdot(1-\varepsilon) n \mu \\
& \geq\left[P_{\mathbf{0}, \mathbf{n}}\left(\neg A_{m}^{1}\right)-\varepsilon\right] \cdot(1-\varepsilon) n \mu .
\end{aligned}
$$

By symmetry, $P_{\mathbf{0}, \mathbf{n}}\left(\neg A_{m}^{1}\right)=P_{\mathbf{0}, \mathbf{n}}\left(\neg A_{m}^{2}\right)=P_{\mathbf{0}, \mathbf{n}}\left(\neg A_{m}\right) / 2$, and, using the fact that $P_{\mathbf{0}, \mathbf{n}}\left(A_{m}\right)<1 / 2$, it follows that $P_{\mathbf{0}, \mathbf{n}}\left(\neg A_{m}^{1}\right) \geq 1 / 4$. Thus

$$
\mathrm{E}\left[\mathbf{1}_{\left\{\neg A_{m}^{1}\right\}} T_{\mathbf{0 , n} \mathbf{n}}\right] \geq\left(\frac{1}{4}-\varepsilon\right)(1-\varepsilon) n \mu,
$$

which in conjunction with (13) and (14) yields the estimate 


$$
\begin{aligned}
\mathrm{E}\left[T_{\mathbf{n},-\mathbf{m}}-T_{\mathbf{0},-\mathbf{m}}\right] & \leq \mathrm{E}\left[T_{\mathbf{0}, \mathbf{n}}\right]-\mathrm{E}\left[\mathbf{1}_{\left\{\neg A_{m}^{1}\right\}} T_{\mathbf{0}, \mathbf{n}}\right] \\
& \leq(1+\varepsilon) n \mu-\left(\frac{1}{4}-\varepsilon\right)(1-\varepsilon) n \mu \\
& \leq\left(\frac{3}{4}+3 \varepsilon\right) n \mu .
\end{aligned}
$$

Now, if $\varepsilon$ is small, clearly $(3 / 4+3 \varepsilon)<(1-2 \varepsilon)$. Hence, to sum up, assuming that $P_{\mathbf{0}, \mathbf{n}}(A)=0$, we have showed that, if $\varepsilon$ is small and $m$ is large, then $\mathrm{E}\left[T_{\mathbf{n},-\mathbf{m}}-T_{\mathbf{0},-\mathbf{m}}\right]<(1-2 \varepsilon) n \mu$. But this contradicts the fact that (12) should hold for arbitrarily large $m$ and consequently we must have $P_{\mathbf{0}, \mathbf{n}}(A)>0$.

\section{References}

Deijfen, M. (2003): Asymptotic shape in a continuum growth model, Adv. Appl. Prob. 35, 303-318.

Deijfen, M. and Häggström, O. (2003): The initial configuration is irrelevant for the possibility of mutual unbounded growth in the two-type Richardson model, Comb. Prob. Computing, to appear.

Deijfen, M., Häggström, O. and Bagley, J. (2003): A stochastic model for competing growth on $\mathbb{R}^{d}$, Markov Proc. Relat. Fields 10:2, 217-248.

Garet, O. and Marchand, R. (2004): Coexistence in two-type first-passage percolation models, Ann. Appl. Prob., to appear.

Häggström, O. and Pemantle, R. (1998): First passage percolation and a model for competing spatial growth, J. Appl. Prob. 35, 683-692.

Häggström, O. and Pemantle, R. (2000): Absence of mutual unbounded growth for almost all parameter values in the two-type Richardson model, Stoch. Proc. Appl. 90, 207-222. 